\title{
Ultrastructural changes produced in rheumatoid synovial membrane by chrysotherapy
}

\author{
F. N. GHADIALLY, A. F. ORYSCHAK, AND D. M. MITCHELL \\ From the Department of Pathology and the Rheumatic Disease Unit, University Hospital, University of \\ Saskatchewan, Saskatoon, Saskatchewan, S7NOWO, Canada
}

\begin{abstract}
Ghadially, F. N., Oryschak, A. F., and Mitchell, D. M. (1976). Annals of the Rheumatic Diseases, 35, 67-72. Ultrastructural changes produced in rheumatoid synovial membrane by chrysotherapy. Biopsies of rheumatoid synovial membrane before and after chrysotherapy were examined with the light and electron microscopes, and subjected to electronprobe $x$-ray analysis. The main change noted was the occurrence of electron-dense deposits of characteristic morphology within pre-existing lysosomes. Such deposits were also seen in other varieties of lysosomal bodies. $X$-ray analysis showed the presence of gold, phosphorus, osmium, and calcium in these deposits. The present study shows that gold enters the pre-existing lysosomes, supporting the concept that the beneficial effects of chrysotherapy are due to its action on the lysosome.
\end{abstract}

Electron microscope studies have now unequivocally demonstrated a remarkable increase in the lysosome population in the rheumatoid synovial membrane (Barland, Novikoff, and Hamerman, 1964; Norton and Ziff, 1966; Wyllie, Haust, and More, 1966; Ghadially and Roy, 1967). It has been suggested that perpetuation of the inflammatory process and the ensuing destruction of articular tissues are due to the release of hydrolytic enzymes either by seepage or by rupture of lysosomes (Lehman, Krearn, and Brogna, 1964; Ghadially and Roy, 1969; Lack, 1969).

It has also been pointed out that many important agents used in the treatment of chronic inflammatory joint diseases, such as glucocortoids, chloroquine, and colchicine, interfere with the function or release of substances from lysosomes by stabilizing the lysosomal membrane. Conversely, agents such as streptolysin $\mathbf{S}$ and filipin which labilize the lysosomal membrane produce inflammation, arthritis, and cartilage destruction when injected into the joint (Weissmann and Thomas, 1964; Weissmann, 1965, Weissmann and others, 1965; Pras and Weissmann, 1966).

It has been suggested that gold salts might also act by stabilizing the lysosomal membrane, but there is no evidence to support this. Persellin and Ziff (1966) have, however, shown that gold inhibits lysosomal enzymes of macrophages and they have conse-

Accepted for publication July 19, 1975.

Correspondence Dr. F. N. Ghadially. quently suggested that a similar mechanism might also operate in the synovial membrane.

It is currently thought that the therapeutic effect of gold lies in its probable action on the lysosome. The possibility that morphological alterations in the lysosome population in rheumatoid synovial membrane and other ultrastructural changes may occur as a result of chrysotherapy and might be shown by electron microscopy led us to study rheumatoid synovial membrane before and after chrysotherapy. In this paper we show that gold administered intramuscularly does indeed find its way into pre-existing lysosomes in the synovial membrane and that its presence can be shown unequivocally by electron probe $x$-ray analysis, which permits the atomic analysis of small areas within the cell.

Gold has now been shown in the lysosomal bodies which form in the chondrocytes of rabbit articular cartilage after intra-articular injection of sodium aurothiomalate (Myocrisin). Such bodies have been called 'aurosomes' (Oryschak and Ghadially, 1974), and the term will be used for the gold-containing lysosomes described in this paper.

\section{Materials and methods}

PATIENTS AND SPECIMENS

Ten patients ( 3 males, 7 females), ranging in age from 16 to 61 years, with clinically established rheumatoid arthritis ( 3 months to 10 years' duration) defined as 
classical or definite by the A.R.A. criteria (Rodnan, McEwen and Wallace, 1973), were included in this study. A percutaneous biopsy of synovial membrane was obtained with the Parker-Pearson needle both before and after chrysotherapy. However, not all attempts at biopsy were successful in each patient, for at times only fibrinous material was obtained, or the sample was inadequate for light and electron microscope study.

The material and patients studied were divided into three groups. (1) 5 patients with successful pretreatment biopsies, but unsuccessful post-treatment biopsies; (2) 3 patients with successful pretreatment and post-treatment biopsies; (3) 2 patients with only post-treatment biopsies. Thus a total of 8 specimens of untreated rheumatoid synovial membranes and 5 specimens of membranes obtained after chrysotherapy were studied.

\section{TREATMENT REGIMEN}

Gold in the form of sodium aurothiomalate was given to patients with rheumatoid arthritis by weekly intramuscular injections. In order to avoid anaphylactic reactions the initial dose was $10 \mathrm{mg}$, the second dose $25 \mathrm{mg}$, and then $50 \mathrm{mg}$ if there were no untoward effects. The initial course of therapy lasted 5 months by which time a total of approximately $1 \mathrm{~g}$ of gold had been given. The posttreatment biopsy was collected at this time. A maintenence dose of $50 \mathrm{mg}$ monthly was then given for an additional 6 or 7 months. In all cases reported here there was both subjective and objective clinical improvement. No untoward effects or complications developed in any of the patients as a result of needle biopsy.

\section{PREPARATION OF TISSUES}

For the light microscope specimens were fixed in neutral $10 \%$ formalin, processed in the usual way, and routine haematoxylin-eosin stained sections were prepared. For the electron microscope tissues were fixed in 3\% glutaraldehyde in cacodylate buffer, $\mathrm{pH} \mathbf{7 \cdot 3}$, for 1 hour and postfixed in $2 \%$ osmium tetroxide in cacodylate buffer, pH 7·3, for 1 hour. The fixed tissues were then dehydrated in increasing concentrations of ethanol cleared in propylene oxide and embedded in Epon. Ultrathin sections approximately $60 \mathrm{~nm}$ thick were mounted on copper grids, stained with uranium acetate and lead hydroxide, and examined with the the Zeiss EM-9S electron microscope.

\section{X-RAY ANALYSIS}

Further sections $100-150 \mathrm{~nm}$ thick were prepared from the Epon blocks for electron probe $x$-ray analysis using a scanning electron microscope (Cambridge Stereoscan) in the transmission mode and an energy dispersive $x$-ray analysis system coupled to a computer (Edax-Edit system). These sections were examined either unstained or stained with uranium only.

The method of $x$-ray analysis was as follows. A grid bearing a section was placed in a stage specially adapted to give a transmission image with the scanning electron microscope. When an area of interest (target) such as a lysosome suspected of containing gold was located, the scanning beam was stopped and adjusted to lie within the centre of the target. $X$-ray counts were then collected for a period of 200 seconds live time. The spectrum thus obtained was displayed on a cathode-ray screen and the counts stored in a memory bank of the computer. Simi- larly, a spectrum was also obtained from the background that is to say from an adjacent area of cell cytoplasm, and stored in another memory bank. The final spectrum showing the elemental composition of the target area was obtained by subtracting the background spectrum from the target spectrum after smoothing and properly normalizing the background spectrum from the cytoplasm to the same background level as the spectrum from the target. The final spectrum was then displayed on the cathode-ray screen and recorded photographically.

\section{Results}

\section{LIGHT MICROSCOPE}

The amount of material available for histological analysis from the needle biopsies was disappointingly small. We do not know whether these are representative samples or not. However, a comparison of the pretreatment and post-treatment biopsies showed no consistent change except that the cellular inflammatory exudate seemed to be less marked in the post-treatment biopsies. Hypertrophic, atrophic, and fibrosed examples of synovial membranes were found in both groups, confirming the duration of disease from 3 months to 10 years in this group of patients. No consistent difference in any other parameter such as the amount of fibrinous material or haemosiderine present was evident in these two groups of biopsies.

\section{ELECTRON MICROSCOPE}

The pretreatment biopsies showed the now wellknown ultrastructural features of the rheumatoid synovial membrane (for review see Ghadially and Roy, 1969), such as an increase in the population of type $B$ and intermediate cells, intracytoplasmic filaments and lysosomes. Such changes were also present in post-treatment biopsies.

Indeed the only consistent characteristic changes which could be attributed to chrysotherapy were seen in the lysosome population. The lysosomes in the rheumatoid synovial membrane (pretreatment) are pleomorphic, yet many of them have a characteristic morphology (Fig. 1), in that they contain a finely granular medium density matrix in which are set a variable number of rounded osmiophilic granules or droplets, presumably lipid in nature. When such droplets are few in number they show a characteristic distribution in that they are usually aligned along the periphery of the lysosome.

In the post-treatment biopsies the lysosomes of the above-mentioned morphology contained, in addition to their usual contents, characteristic highly electron-dense material in the form of fine particles, spicules, and curled formations (Fig. 2). Such structures were never found in the pretreatment biopsy specimens. Transitional forms were abundant, suggesting that these characteristic highly electron- 


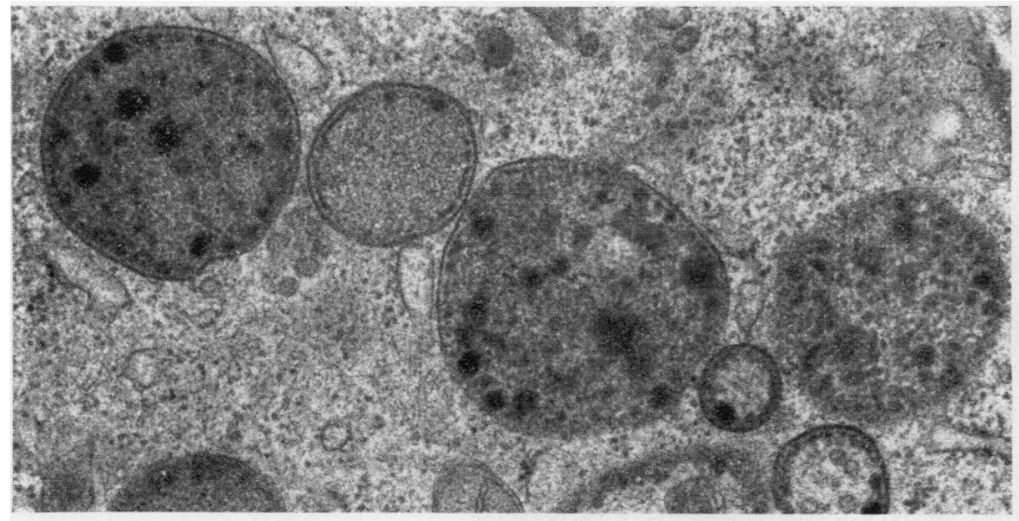

FIG. 1 Lysosomes found in a synovial intimal cell from a pretreatment specimen. Note the osmiophilic lipid droplets set in a granular medium density matrix. $\times 33000$

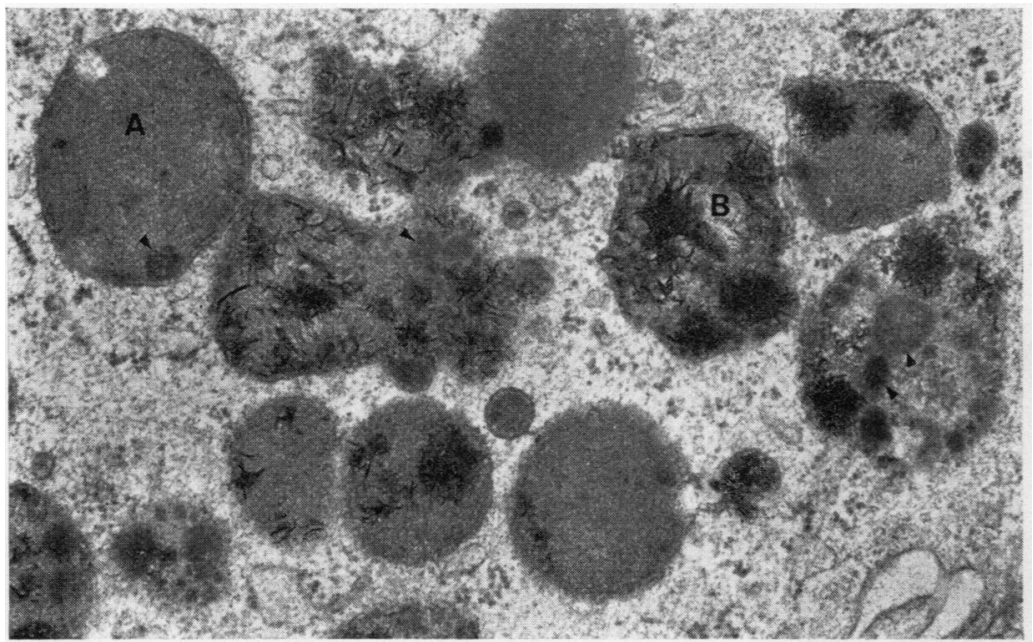

FIG. 2 Synovial intimal cell from a post-treatment specimen showing transitional forms between lysosomes containing no gold deposits (A) and those loaded with electron-dense, rod-like, and curled formations $(B)$ indicating the presence of gold. Note also the presence of numerous lipid droplets (arrowheads). $\times 33000$

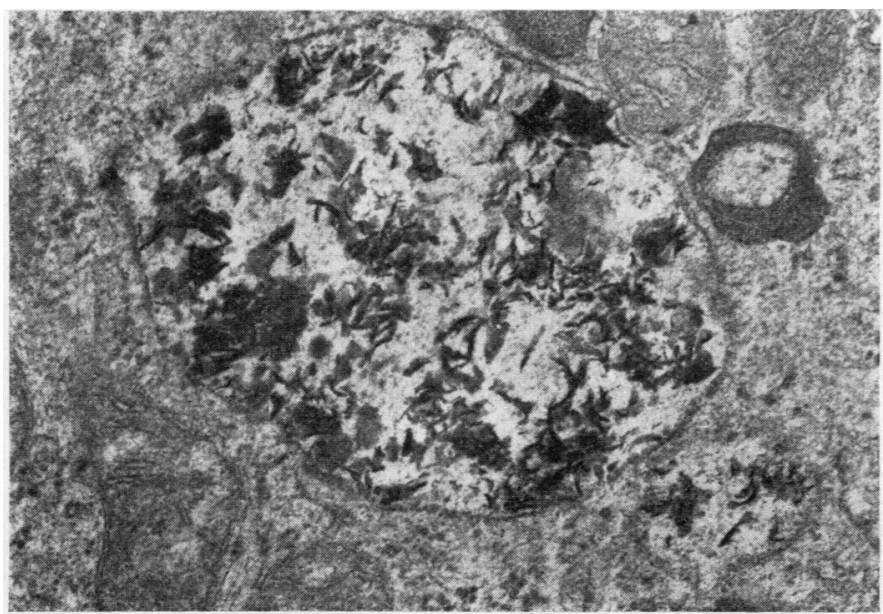

FIG. 3 Aurosome found in a synovial intimal cell after chrysotherapy showing electrondense structures representing gold, set in a lucent matrix. Compare with Fig. 6. $\times 40000$ 


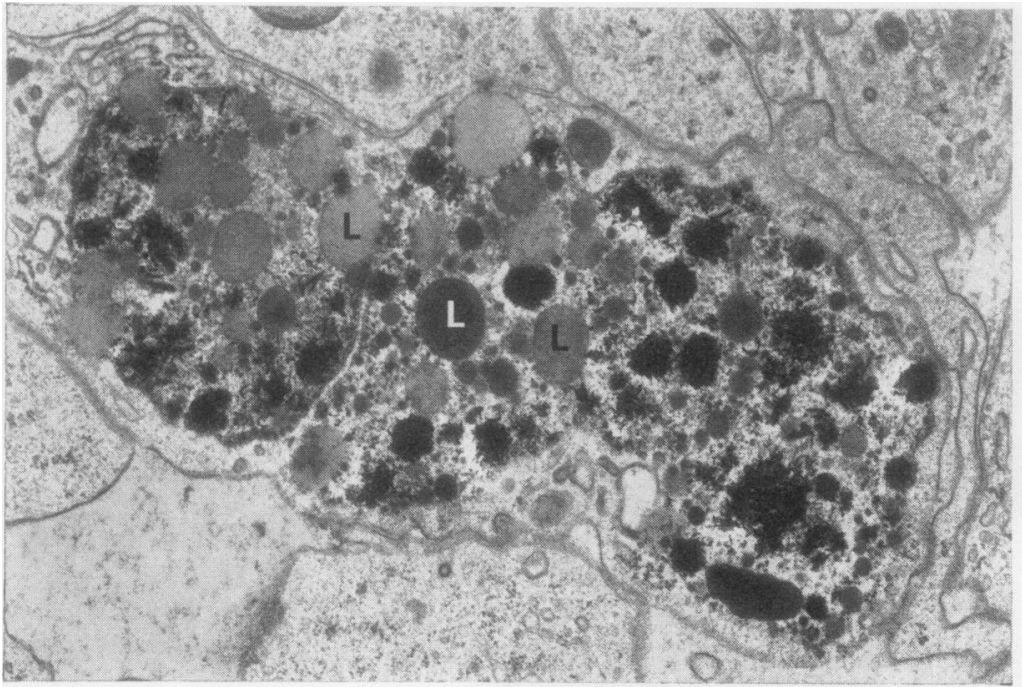

FIG. 4. A large aurosome presumably formed by the fusion of smaller aurosomes. Note lipid droplets $(L)$ ? of varying osmiophilia and the characteristic electron-dense contents. $\times 17000$

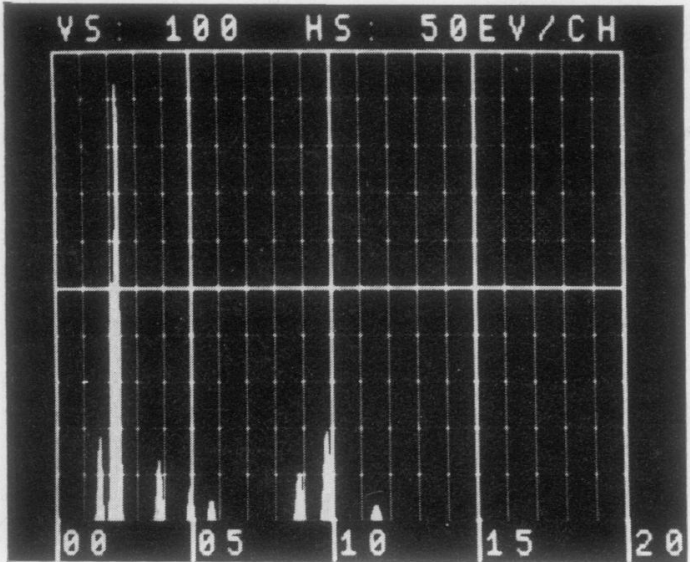

FIG. $5 X$-ray spectrum of an aurosome

dense deposits were developing in pre-existing lysosomes as a result of chrysotherapy. Rare lysosomes of a somewhat different morphology were also seen which contained the characteristic electrondense deposits (attributable to the presence of gold) set in an electron lucent matrix (Fig. 3). Another infrequent finding was the occurrence of large lysosomal bodies containing numerous lipid droplets of varying osmiophilia and the characteristic electrondense deposits attributable to gold (Fig. 4).

X-RAY ANALYSIS

Approximately six aurosomes from each of the 5 post-treatment biopsies were subjected to electronprobe $x$-ray analysis. In each instance when the aurosome was large enough and dense enough (20 instances) we were able to obtain the characteristic gold peaks ( $\mathrm{M}$ at $2.1 \mathrm{KeV}, \mathrm{L} \alpha$ at $9.7 \mathrm{KeV}$, and $\mathrm{L} \beta$ at $11.5 \mathrm{KeV}$ ) as shown in Fig. 5. Smaller peaks indicating the presence of phosphorus ( $\mathrm{K} \alpha$ at $2 \mathrm{KeV}$ ), osmium $(\mathrm{M}$ at $1.9 \mathrm{KeV})$, and traces of calcium $(\mathrm{K} \alpha$ at 3.7 o $\mathrm{KeV}$ ) were also present. In addition, sporadic residual peaks attributable to parts of the instrument, such as aluminium from the scintillator, silicon from the detector, iron and chromium from the microscope chamber were occasionally seen. For example, in Fig. 5 a residual peak at $1.5 \mathrm{KeV}$ representing the $\mathbf{K} \alpha$ of aluminium is present. Such peaks may not be completely cancelled out by subtracting the background spectrum because the amount of electron scattering from the sample (which produces $x$-rays from the microscope hardware) would be expected 


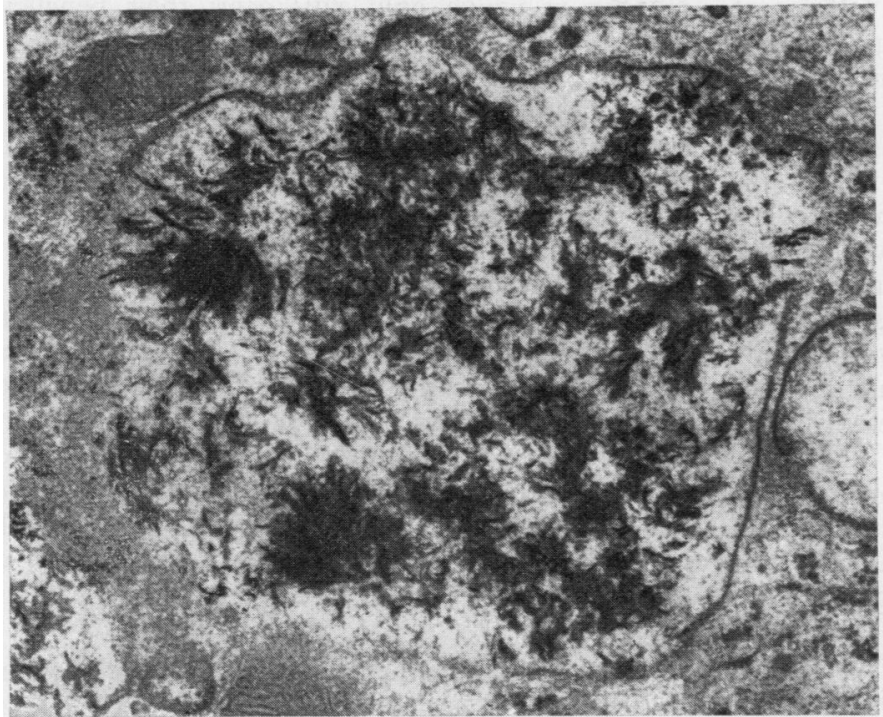

FIG. 6 An aurosome (of a morphology similar to that in Fig. 3) which developed in a rabbit synovial intimal cell 3 days after intra-articular injection of $25 \mathrm{mg}$ sodium aurothiomalate. $\times 40000$

to be enhanced because of the presence of heavy elements such as gold and osmium in the aurosome.

\section{Discussion}

\section{AUROSOMES}

The results show that aurosomes characterized by the presence of highly dense rod-like and curled formations develop in rheumatoid synovial membrane after chrysotherapy. In a previous study on the aurosomes that develop in the chondrocytes after intra-articular injection of gold salts it was shown (using unosmicated and unstained specimens) that these rod-like and curled formations represent sections through compacted membranes studded with very fine particles which presumably represent gold. (See Fig. 3, Oryschak and Ghadially, 1974.) The structure in the human material is similar but cannot be shown because unosmicated material was not collected. The aurosomes that develop in the chondrocytes and in the synovial membrane (unpublished observation) in the rabbit after intra-articular injection of sodium aurothiomalate contain little besides the characteristic rod-like and curled structures set in an electron lucent matrix (Fig. 6). Although rare examples of such presumably de novo aurosomes were seen (Fig. 3), the bulk of the aurosomes seems to have developed by the deposition of gold in pre-existing lysosomes (Fig. 2).

The development of the very large aurosomes (Fig. 4) is not clear but may be due to the fusion of aurosomes; the situation here being analogous to that seen in chronic haemarthrosis where evidence of fusion of siderosomes and inclusion of other structures such as mitochondria and lipid droplets into such structures has been noted (Roy and Ghadially, 1969).

X-RAY ANALYSIS

Atomic analysis permits the identification of elements present but gives no indication of the nature of the compounds formed from such elements. Our results provide unequivocal proof of the presence of gold here, for quite high peaks for gold were found on analysis of aurosomes. It is safe to say that there is also a significant amount of osmium and phosphorus present in the aurosomes and that this probably reflects the presence of osmiophilic lipids and phospholipid membranous material. The significance of the traces of calcium present remains obscure. Previous suggestions as to the nature of gold deposits (in other sites) produced by soluble gold salts has been that gold may occur in metallic form or as a sulphide (Koppenhofer, 1936). In our case we can rule out the 'sulphide' as no sulphur was detected but the suggestion that gold occurs in a metallic form is consistent with our findings.

\section{CHR YSOTHER APY}

Although the beneficial effects of chrysotherapy in the treatment of rheumatoid arthritis are widely recognized, it is not clear how or why gold exerts such an effect. The idea that the beneficial effects may be due to alterations in the immunological mechanisms is not supported by Persellin and others (1967), who found that gold inhibits neither circulating antibody production nor delayed hypersensitivity. On the other hand, as noted in the introduction, the suggestion that gold may exert its beneficial effect by 
inactivating lysosomal enzymes has much to support it. However, until now evidence supporting the idea that parentrally administered gold does in fact enter the lysosomes in the rheumatoid synovial membrane has not been forthcoming. Indeed animal experiments show that when sodium aurothiomalate is administered intra-articularly, fresh lysosomal bodies containing gold deposits develop in synovial intimal cells and in chondrocytes of articular cartilage (Norton, Lewis, and Ziff, 1968; Oryschak and Ghadially, 1974; unpublished observations). If this were to happen in the rheumatoid synovium it would only add to the burden of lysosomes present in the tissue.

However, the situation in the rheumatoid synovial membrane is somewhat different, for only rare examples of newly formed aurosomes (as found in experimental animals) were encountered, and the bulk of the gold deposits in the synovial membrane were localized in pre-existing lysosomes. This finding then clearly supports our concepts regarding the role of lysosomes in rheumatoid arthritis and the probable manner in which gold salts exert their beneficial effect.

How or why gold salts locate within pre-existing lysosomes was not shown by our study but the observation that metals can do this is not new. For $\overrightarrow{\vec{F}}$ example, Ericsson (1969) has shown that in Thorotrast- or iron-treated rats the peribillary dense bodies (i.e. pre-existing lysosomes of rat liver) become loaded with these metals.

Lewis and Ziff (1966) have found that no significant histological alteration occurs in the synovial mem- के brane after chrysotherapy. Our findings agree with $\vec{\nabla}$ this except that we did note a reduction in the inflammatory cell infiltrate although the samples of tissue examined were small. However, if such an anti-inflammatory effect does indeed occur it would be easily explained on the basis of inactivation of lysosomal enzymes by gold salts.

This work was supported by grants from the Canadian Arthritis and Rheumatism Society and the Medical Research Council of Canada.

\section{References}

Barland, P., Novikoff, A. B., And Hamerman, D. (1964) Amer. J. Path., 44, 854 (Fine structure and cytochemistry of the rheumatoid synovial membrane, with special reference to lysosomes)

EricsSON, J. L. E. (1969) 'Mechanism of cellular autophagy' in 'Lysosomes in Biology and Pathology', ed. J. T. Dingle and H. B. Fell, p. 345. North-Holland, Amsterdam and London

Ghadially, F. N., AND Roy, S. (1967) Ann. rheum. Dis., 26, 426 (Ultrastructure of synovial membrane in rheumatoid arthritis)

- - (1969) In 'Ultrastructure of Synovial Joints in Health and Disease'. Butterworths, London

KopPenhoffer, G. F. (1936) Dtsch. med.Wschr., 25, 1011 (Ablagerung und Verteilung von Gold nach Zulphur Anorganischer und organischer Goldpräparate)

LACK, C. H. (1969) 'Lysosomes in relation to arthritis' in 'Lysosomes in Biology and Pathology', ed. J. T. Dingle and H. B. Fell, p. 493. North-Holland, Amsterdam and London

Lehman, M. A., Kream, J., And Brogna, D. (1964) J. Bone Jt Surg., 46A, 1732 (Acid and alkaline phosphatase activity in serum and synovial fluid of patients with arthritis)

LEWIS, D. C., AND ZIFF, M. (1966) Arthr. and Rheum., 9, 682 (Intra-articular administration of gold salts)

NortON, W. L., AND ZIFF, M. (1966) Ibid., 9, 589 (Electron microscopic observations on the rheumatoid synovial membrane)

-—, LEwIS, D. C., AND ZIFF, M. (1968). Ibid., 11, 436 (Electron-dense deposits following injection of gold sodium thiomalate and thiomalic acid)

Oryschak, A. F., and Ghadially, F. N. (1974) Virchows Arch. Abt. B., 17, 159 (Aurosomes in rabbit articular cartilage)

Persellin, R. H., AND Ziff, M. (1966) Arthr. and Rheum., 9, 57 (The effect of gold salt on lysosomal enzymes of the peritoneal macrophage)

- HESS, E. V., AND ZIFF, M. (1967) Ibid., 10, 99 (Effect of gold salt on the immune response)

Pras, M., and Weissmann, G. (1966) Ibid., 9, 533 (Abst.) (Filipin-induced arthritis)

Rodnan, G. P., McEwen C., And Wallace, S. L. (1973) J. Amer. med. Ass., 224, 661 (Primer on the rheumatic diseases)

Roy, S., ANd Ghadially, F. N. (1969) Ann. rheum. Dis., 28, 402 (The synovial membrane in experimentally produced chronic haemarthrosis)

WeissmanN, G. (1965) New Engl.J. Med., 273, 1084 (Lysosomes)

_- , and Thomas, L. (1964) Recent Progr. Hormone Res., 20, 215 (Effects of corticosteroids on connective tissue and lysosomes)

- - Becher, B., Wiedermann, G., ANd Bernheimer, A. W. (1965) Amer. J. Path., 46, 129 (Studies on lysosomes. VII. Acute and chronic arthritis produced in rabbits by intra-articular injections of streptolysin S)

Wyllie, J. C., Haust, M. D., AND More, R. H. (1966) Lab. Invest., 15, 519 (The fine structure of synovial lining cells in rheumatoid arthritis) 